\begin{tabular}{|c|c|c|}
\hline 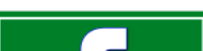 & Int.J.Curr.Microbiol.App.Sci (2021) 10(08): 578-584 & \\
\hline & $\begin{array}{l}\text { International Journal of Current Microbiology and Applied Sciences } \\
\text { ISSN: 2319-7706 Volume } \mathbf{1 0} \text { Number } 08 \mathbf{( 2 0 2 1 )} \\
\text { Journal homepage: } \underline{\text { http://www.ijcmas.com }}\end{array}$ & 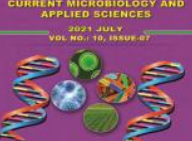 \\
\hline $\begin{array}{l}\text { EXCELLENT } \\
\text { PUBLISHERS }\end{array}$ & & \\
\hline
\end{tabular}

\title{
Staphylococcal Positivity and Sensitivity in Invasive Bloodstream Infections using Automated Bactec in Tertiary Care Hospital of North India
}

\author{
Pankaj Katoch $^{1 *}$, Ambika Sood ${ }^{2}$ and Surinder Singh ${ }^{2}$ \\ ${ }^{1}$ Department of Microbiology, ${ }^{2}$ Department of Paediatrics, IGMC, Shimla, India \\ *Corresponding author
}

\begin{tabular}{|l|}
\hline Ke y w o r d s \\
Blood Stream \\
Infections, MRSA, \\
Staphylococcal \\
Positivity, and \\
Sensitivity, \\
Increasing \\
Antibiotic \\
Resistance \\
\hline Article Info \\
\hline Accepted: \\
25 July 2021 \\
Available Online: \\
10 August 2021 \\
\hline
\end{tabular}

\section{A B S T R A C T}

Staphylococcus aureus is present in the anterior nares of about 20-30\% human population. It also resides in the skin, pharynx, acne secretions, and urinary tract also. It can cause a wide spectrum of diseases from fulminant septicemia to localized infections. All the blood culture samples received in the Department of Microbiology for culture by Bactec Bdfx from July 2015 to June2016 were included in the study. The blood culture was observed in the BactecBdfx system for at least 5 days before they are reported as sterile. Among the total 1275 cultures which were positive for bacteria, 344(26.98\%) were of Gram-positive bacteria. Among the total of 344 culture isolates positive for Gram-positive bacteria, Staphylococcus aureus was isolated in 184(53.49\%) cultures, Among the total 184 cultures, maximum staphylococcal isolates were found in the age group of $19-45$ years $(26.63 \%)$ followed by $0-1$ year $(24.46 \%)$. All the Staphylococcus aureus isolates showed $100 \%$ sensitivity to Vancomycin and Linezolid. $90.0 \%$ isolates were sensitive to Aztreonam, Ampicillin Sulbactam(86.67\%), Amikacin $(83.33 \%)$, Tobramycin (83.33\%), co-trimoxazole(78.80\%) and gentamycin (76.08\%). S.aureus showed high-level resistance to Penicillin (66.30\%), Erythromycin (38.58\%), Clindamycin (38.04\%), and Ciprofloxacin (30.98\%). Staphylococcus aureus was the commonest organism among GPC isolates. S.aureus was the most common isolated organism in the adults and infant group. Staphylococcus aureus was maximum sensitive to Vancomycin and Linezolid while maximum resistant to penicillin.

\section{Introduction}

Blood cultures are the most valuable tool used for the diagnosis of bloodstream infections (BSI) and are considered to be the Gold standard for their diagnosis. Hence, the present study is designed to determine the incidence of Staphylococcal positivity and sensitivity in invasive bloodstream infections using automated Bactec systems. The accurate identification of blood isolates and their prompt treatment as per sensitivity report and 
identification of the route of invasion so that appropriate measures initiated well in time are key to the exact management of BSI. ${ }^{1}$

Blood cultures, used for the detection of presumptive viable pathogens, have the advantage of allowing the evaluation of their antimicrobial susceptibility feature; which is not rivaled by any other diagnostic tool available to date.

This is quite important, as some studies have shown that inappropriate empirical therapy is an independent risk factor for treatment failure for severely ill patients with serious lifethreatening infections.

In the most serious cases of sepsis and septic shock the risk of mortality increases by the hour if appropriate antimicrobial therapy is not initiated well in time. ${ }^{1,2,3}$

Indira Gandhi Medical College(IGMC) Shimla is a Tertiary care hospital and patients with above mentioned clinical conditions are treated both in the outpatient department(OPD)and inpatient department (IPD) and usually require blood culture to establish the etiological diagnosis.

Previously no study has been done in IGMC Shimla using an automated Bactec BD FX machine for blood culture.

Hence, the present study is conducted to determine the incidence of Staphylococcal positivity and sensitivity in Invasive bloodstream infections using automated Bactec systems.

The main aim and objectives of this study to determine the incidence of Staphylococcal positivity and sensitivity in Invasive bloodstream infections using automated Bactec systems.

\section{Materials and Methods}

\section{Study Design}

Prospective observational study

\section{Study Setting}

Department of Microbiology, Indira Gandhi Medical College And Hospital, Shimla

\section{Study Period}

one year from July 2015 to June2016

\section{Inclusion criteria}

All the blood culture samples received in the department of microbiology for blood culture by BactecBdfx system.

Patients willing for study.

Blood cultures from all age groups

\section{Exclusion criteria}

Patient not willing to study.

Blood cultures showing mixed growth

All the blood culture samples received in the Department of microbiology for culture by BactecBdfx were included in the study. The blood culture was observed in the BactecBdfx system for at least 5 days before they are reported as sterile.

The sample to be tested is inoculated into the Bactec ${ }^{\text {tm }}$ plus aerobic/f culture bottle for adults and Bactec ${ }^{\mathrm{tm}}$ Peds plus/f for children which is then inserted into the BdBactec fluorescent series instrument for incubation. Each bottle has a sensor that detects an increase in co2 produced by the growth of microorganisms. The sensor monitors every 
10 minutes for an increase in its fluorescence, which is proportional to the amount of $\mathrm{co} 2$ present. A positive reading indicates the presumptive presence of viable microorganisms in the bottle. The positive bottle will be subcultured on Blood agar and MacConkey's agar plates. Following the subculture on solid media from each positive bottle a smear will be prepared for gram staining from that blood culture bottle. The Gram-stained smear will be examined for the presence of microorganisms and presumptive report conveyed to respective departments. The blood agar and Mac Conkey agar plates will be incubated aerobically at $37^{\circ} \mathrm{c}$ for 24 to $48 \mathrm{hrs}$ and then observed for the growth of bacteria. All bacterial isolates will be identified using standard biochemical identification methods. Staphylococcus aureus ATCC 2592 was inoculated along with the test organisms. All the positive isolates were stocked.

\section{Statistical analysis}

The data was analyzed using statistical analysis-epi info7. The data collected was entered into a spreadsheet. The data was checked for any missing values and completed. Analysis in terms of demographic variables, positivity in the processed samples, type of species prevalent, was done using statistical software epi-info version 7(7.1.1.0).

\section{Results and Discussion}

Staphylococcus aureus most commonly causes infections that are difficult to treat at home. So, it is advisable to have a culture and antibiotic susceptibility report done before the initiation of empirical treatment.

In the present study, among the total of 5473 samples suspected of BSI's received in the Department of Microbiology, IGMC, Shimla 1441 were positive. Among the total positive culture, $1275(88.48 \%)$ were positive for Bacteria while 166(11.52\%) were positive for Fungi. Among the total 1275 cultures which were positive for bacteria, 344(26.98\%) were of Gram-positive bacteria. Among the total of 344 culture positive for Gram-positive bacteria, Staphylococcus aureus was isolated in $184(53.49 \%)$ cultures.

Maximum staphylococcal isolates were found in age group of $19-45$ years $(26.63 \%)$ followed by $0-1$ year $(24.46 \%)$, 46-65 years $(20.65 \%),>66$ years $(16.30 \%)$ and $5.98 \%$ in 25 years and 6-18 years each (Table-1).

All the Staphylococcus aureus isolates showed $100 \%$ sensitivity to Vancomycin and Linezolid. $90.0 \%$ isolates were sensitive to Aztreonam, Ampicillin Sulbactam (86.67\%), Amikacin (83.33\%), Tobramycin (83.33\%), Co-trimoxazole(78.80\%), Gentamycin (76.08\%), Levofloxacin (73.33\%), Ciprofloxacin (66.84\%), AmoxicillinClavulanic acid $(66.67 \%)$ and Clindamycin(64.13\%). S.aureus showed highlevel resistance to Penicillin (66.30\%), Erythromycin (38.58\%), Clindamycin (38.04\%), and Ciprofloxacin (30.98\%).

It is important to continuously update and review the epidemiology of BSIs regarding the antibiotic susceptibility pattern of the pathogens generally encountered, so that prompt treatment of patients may be instituted as the sensitivity pattern can vary from one clinical setting to another. The fact we all have to accept is not how resistance is going to increase but how severe its ramifications will be and how much damage the increased prevalence will cause to humans. The increasing resistance to penicillin and related drugs is mostly due to selective pressure due to increased antibiotic exposure. In the present study, we attempted to determine the incidence of Staphylococcal positivity and antimicrobial sensitivity pattern in Invasive 
bloodstream infections using automated Bactec systems. The results obtained in the present study were analyzed and compared with other studies.

The isolation of (13.05\%) Staphylococcus aureus observed in the present study is contrary to reports from studies in African countries (Meremikwu et al.,) which identified a high incidence of Staphylococcus aureus $(48.7 \%)$ bacteremia.

In the present study, it was found that S.aureus showed $100 \%$ sensitivity to Vancomycin and Linezolid. $90.0 \%$ isolates were sensitive to Aztreonam, Ampicillin Sulbactum(86.67\%), Amikacin (83.33\%), Tobramycin (83.33\%), Co-trimoxazole (78.80\%), Gentamycin (76.08\%), Levofloxacin (73.33\%), Ciprofloxacin (66.84\%), AmoxicillinClavulanic acid (66.67\%) and Clindamycin (64.13\%). S.aureus showed high-level resistance to Penicillin (66.30\%), Erythromycin (38.58\%), Clindamycin (38.04\%), and Ciprofloxacin (30.98\%). Arora $\mathrm{U}$ and Devi $\mathrm{P}$ et al., ${ }^{5}$ in their study also found maximum resistance to Ampicillin (74.61\%) and Erythromycin (69.67\%) amongst the GPC. In their study, Fluit et al., ${ }^{6}$ reported that all Staphylococci remained fully susceptible to Vancomycin. Rahbar M et al., ${ }^{7}$ found S.aureus were resistant to penicillin $(82.6 \%)$ but sensitive to Cotrimoxazole and Vancomycin. MRSA is resistant to B-lactam antibiotics which include Penicillin and Cephalosporins. This leads to the fact that patients with MRSA cannot be treated with readily available drugs such as Penicillin and Cephalosporins. MRSA bacteremia is associated with an increased mortality rate, longer duration of hospital stay, and puts a lot of financial pressure on patients economically or the economy of the country as a whole. MRSA is a greater risk factor for mortality because it is very difficult to treat. (Khairulddin et al., $)^{8}$. The emergence of MRSA will put lots of pressure on health facilities to stock much more expensive drugs such as Vancomycin and Teicoplanin which are currently used in the treatment of MRSA infections. An increased number of cases are being reported nowadays of Vancomycin resistance.

Table.1 Distribution of staphylococcus infection

\begin{tabular}{|c|c|c|}
\hline Serial No. & Age group & Proportion \\
\hline $\mathbf{1 .}$ & $0-1$ year & $45(24.46 \%)$ \\
\hline $\mathbf{2 .}$ & $2-5$ years & $11(5.98 \%)$ \\
\hline $\mathbf{3 .}$ & 6-18years & $11(5.98 \%)$ \\
\hline $\mathbf{4 .}$ & $19-45$ years & $49(26.63 \%)$ \\
\hline $\mathbf{5 .}$ & $46-65$ years & $38(20.65 \%)$ \\
\hline $\mathbf{6 .}$ & $\geq 66$ years & $30(16.30 \%)$ \\
\hline $\mathbf{7 .}$ & Total & $184(100 \%)$ \\
\hline
\end{tabular}

Table.2 Sensitivity and resistance of Staphylococcus aureus

\begin{tabular}{|c|c|c|c|c|c|c|c|c|c|c|c|c|c|c|c|}
\hline Sensitivity & Amika & Amoxiclav & Ampisal & Ampicillin & Aztreon & Cipro & Clinda & Cotri & Eryth & Genta & Levo & Lino & Netli & Tobra & Vanco \\
\hline Sensitive & 50 & 40 & 52 & 62 & 54 & 123 & 118 & 145 & 103 & 140 & 44 & 60 & 38 & 50 & 60 \\
\hline Resistant & 10 & 20 & 8 & 122 & 6 & 57 & 70 & 34 & 71 & 44 & 16 & 0 & 22 & 10 & 0 \\
\hline Intermediate & 0 & 0 & 0 & 2 & 0 & 4 & 0 & 5 & 10 & 0 & 0 & 0 & 0 & & \\
\hline Total & 60 & 60 & 60 & 184 & 60 & 184 & 184 & 184 & 184 & 184 & 60 & 60 & 60 & 60 & 60 \\
\hline
\end{tabular}


Fig.1 Distribution of staphylococcus infection

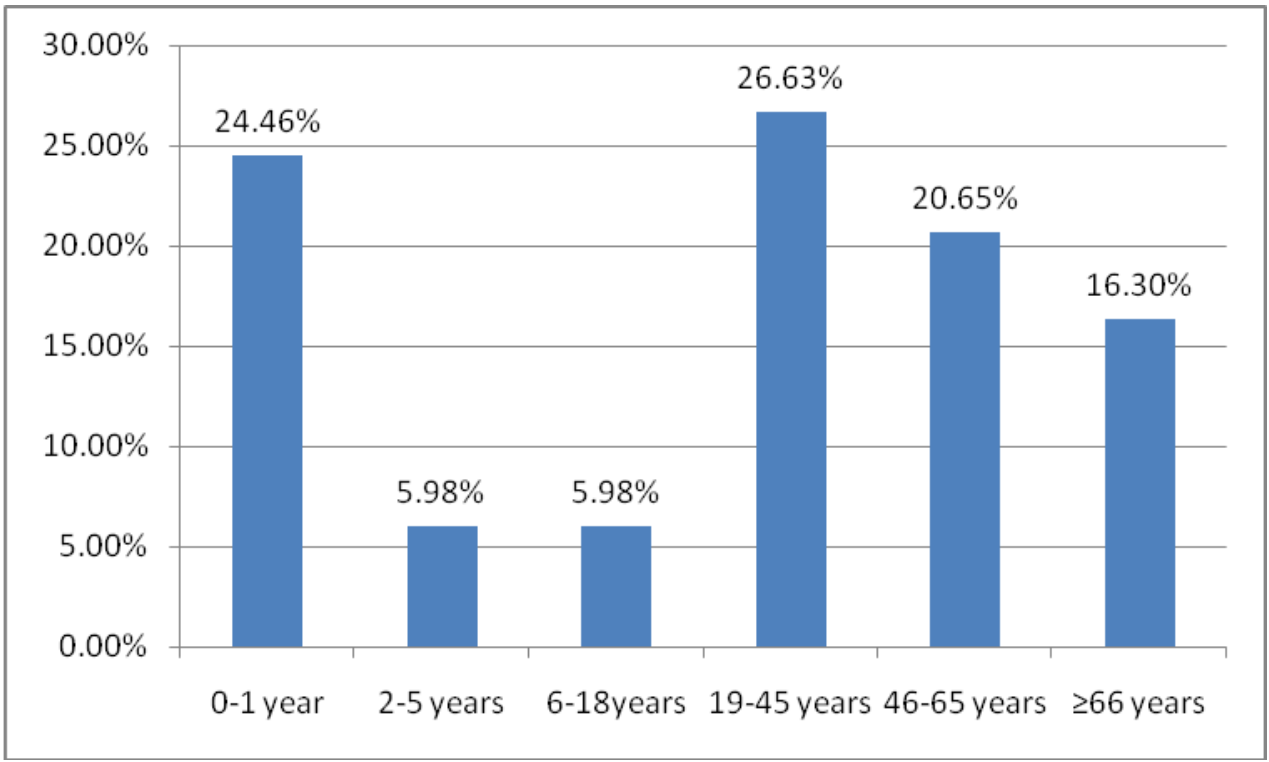

Fig.2 Sensitivity of Staphylococus aureus

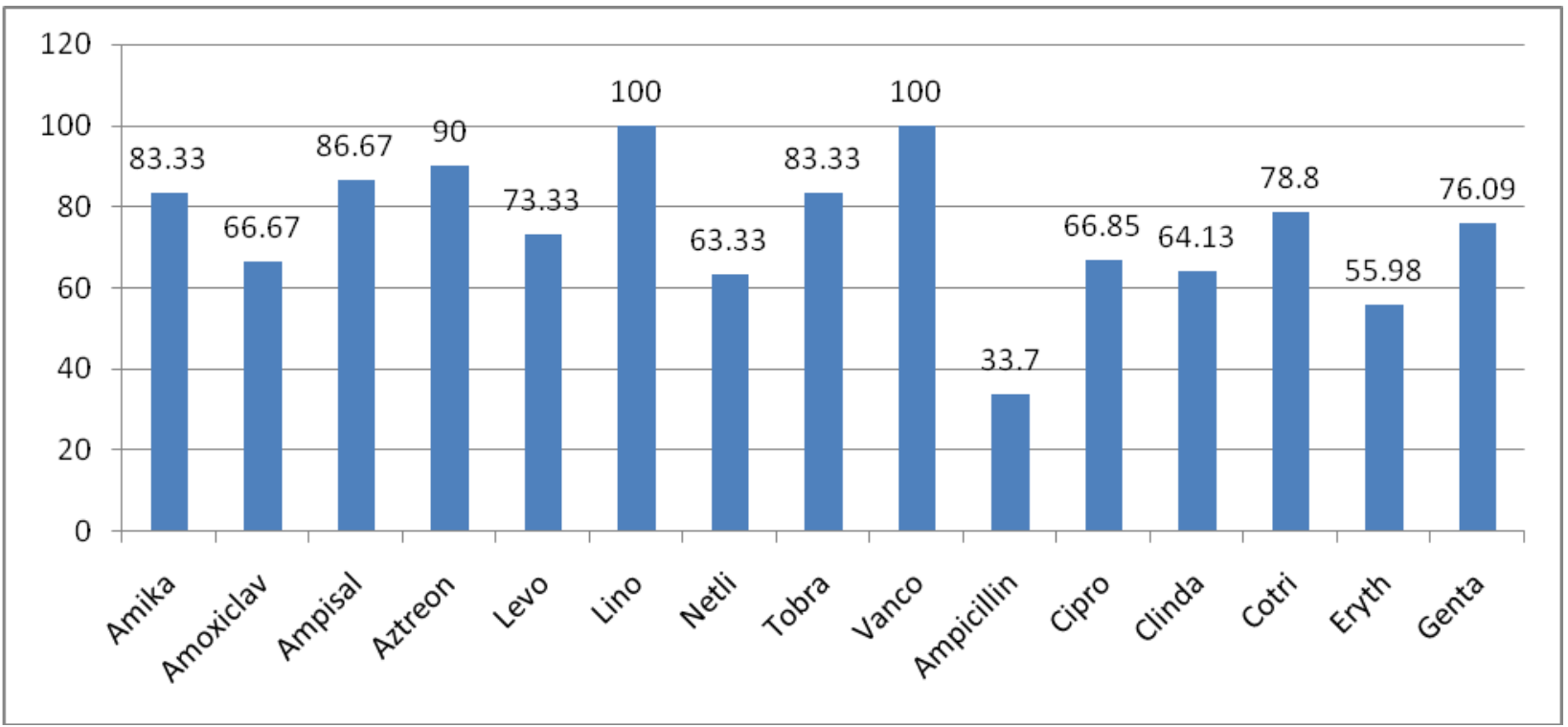


Fig.3 Resistance of Staphylococus aureus

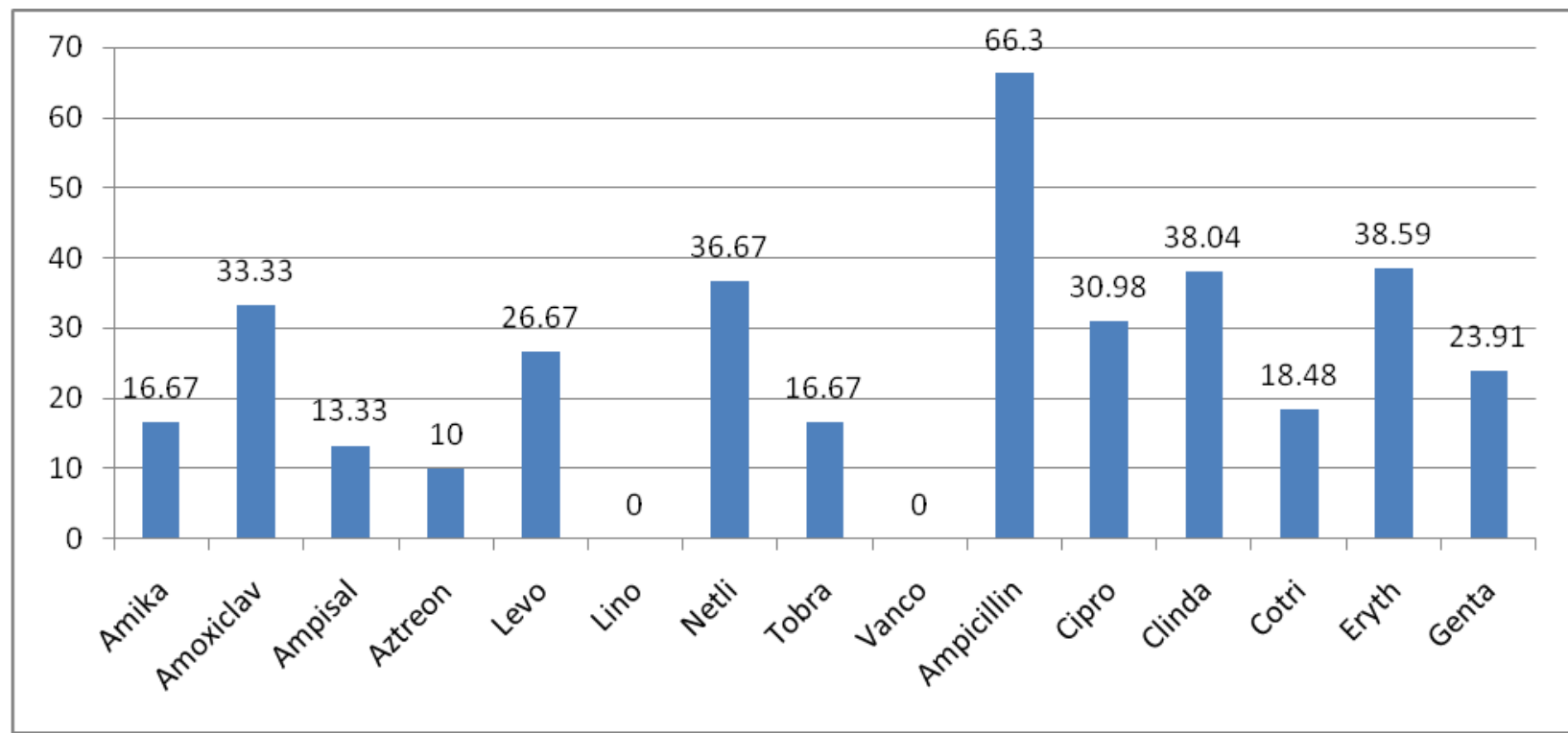

The day is not far when resistance to these high-end antibiotics will be common and we will have nothing in hand because new drug development is a time-consuming and costly procedure. To prevent the spread of MRSA in hospitals, employers should ensure the availability of adequate facilities and supplies that encourage workers to practice good hand hygiene (Khairlddin et al., $)^{8}$. Non-adherence to infection control practices, such as hand hygiene, is the most potentially modifiable cause of healthcare-associated infections in hospitals. $^{8}$

Staphylococcus aureus was the commonest organism among GPC isolates. S.aureus was the most common isolated organism in the adults and infant group. Staphylococcus aureus was maximum sensitive to Vancomycin and Linezolid while maximum resistant to penicillin.

\section{References}

1. Mancini N, Carletti S, Ghidoli N, Cichero P, Burioni R, Clementi M. The era of molecular and other non-culture-based methods in diagnosis of sepsis. ClinMicrobiol

Rev. 2010;23(1):235-251.

2. Peters R P, van Agtmael M A, Danner S A, Savelkoul P H, Vandenbroucke-Grauls C M. New developments in the diagnosis of bloodstream infections. Lancet Infect Dis. 2004 Dec;4(12):751-60.

3. Lamy B, Dargère S, Arendrup M C, Parienti J-J and Tattevin P (2016) How to Optimize the Use of Blood Cultures for the Diagnosis of Bloodstream Infections? A State-of-the-Art. Front. Microbiol. 7:697.

4. Meremikwu M M Bacterial isolates from blood cultures of children with suspected septicemia in Calabar, Nigeria. BMC Infectious Diseases20055:110

5. Arora U, Devi P. Bacterial profile of bloodstream infections and antibiotic resistance pattern of isolates. J. K. Science. 2007;9:186-90.

6. Fluit A C, Jones M E, Schmitz F J, Acar $\mathrm{J}$, Gupta R, Verhoel J, et al., Antimicrobial susceptibility and frequency of occurrence of clinical blood isolates in Europe from the sentry antimicrobial surveillance program, 1197 and 1998. Clin Infect Dis. 2000;30:454- 
60.

7. Rahbar M, Gra-Agaji R, Hashemi S. Nosocomial bloodstream infections in Imam Khomeini hospital, Urmia, Islamic Republic of Iran, 1999-2001. East Mediaterr Health J. 2005;11:478-84.
8. Khairulddin N, Bishop L, Lamagni LT, Sharland M, Duckworth G. Emergence of Methicillin-resistant Staphylococcus aureus (MRSA) bacteremia among children in England and Wales, 19902001. Arch Dis Child 2004; 89: 378-9.

\section{How to cite this article:}

Pankaj Katoch, Ambika Sood and Surinder Singh. 2021. Staphylococcal Positivity and Sensitivity in Invasive Bloodstream Infections using Automated Bactec in Tertiary Care Hospital of North India. Int.J.Curr.Microbiol.App.Sci. 10(08): 578-584.

doi: https://doi.org/10.20546/ijcmas.2021.1008.068 